\title{
Current status of robotic adrenalectomy in the United States
}

\author{
Raymon H. Grogan \\ Section Chief of Endocrine Surgery, Baylor St. Luke's Medical Center, Houston, TX, USA \\ Correspondence to: Raymon H. Grogan, MD, MS, FACS. Associate Professor, 7200 Cambridge Street, $7^{\text {th }}$ Floor, Houston, TX 77030 , USA. \\ Email: rgrogan@bcm.edu.
}

\begin{abstract}
Since its introduction more than 30 years ago, laparoscopic adrenalectomy has become the gold standard approach to adrenal surgery for the majority of adrenal pathology. It has been shown to have superior clinical benefits to open adrenalectomy for the majority of cases of adrenal surgery. Robot-assisted adrenalectomy has also been shown to be a reasonable alternative approach to adrenal gland resection. Even though robot-assisted adrenalectomy has been performed in the United States for more than 20 years, it has yet to become the gold-standard for this operation, evidenced by the fact that in the United States laparoscopic adrenalectomy remains more common than robot-assisted adrenalectomy. Market factors, clinical factors and surgeon factors all play a role in the spread and adoption of robotic surgery in general. Here we review the most up to date literature on the state of robotic adrenalectomy in the United States, and explore some of the factors that may be influencing the rate of adoption of robotic adrenalectomy in the United States.
\end{abstract}

Keywords: Robotic adrenalectomy; laparoscopic adrenalectomy; robotic adrenalectomy trends; trends in robotic surgery, trends in adrenal surgery

Submitted Mar 04, 2020. Accepted for publication Mar 23, 2020.

doi: $10.21037 /$ gs.2020.03.39

View this article at: http://dx.doi.org/10.21037/gs.2020.03.39

Adrenal surgery in the United States, like many other surgical procedures, has shifted over time from an open to a minimally invasive operation. The first laparoscopic adrenalectomy was reported in 1992 by Dr. Gagner in Montreal (1). Shortly after, the first laparoscopic adrenalectomy in the United States was also reported (2). Today laparoscopic adrenalectomy is considered the gold standard for the majority of adrenal pathology (3). The shift from open to laparoscopic adrenalectomy is due to an overwhelming accumulation of evidence over the last three decades showing that laparoscopic adrenalectomy is a safe and effective means of removing the adrenal gland. When compared to open adrenalectomy, laparoscopic adrenalectomy is associated with improved postoperative pain levels, decreased morbidity, shorter hospital stays, quicker return of bowel function, and faster overall recovery (4).

Adoption of the laparoscopic approach for adrenalectomy was not immediate, and it was several years before it was considered a routine and standard operation. It took seven years for 600 laparoscopic adrenalectomy cases to be reported in the literature, and it was around that time that it began to be considered the gold standard for adrenalectomy (3). It was during the transition from open to laparoscopic adrenalectomy, that robotic surgery began in earnest. The Da Vinci robotic system gained FDA approval for general laparoscopic procedures in the United States in the year 2000. By the year 2000 laparoscopic adrenalectomy was considered a routine operation in the United States, and the same year the robot gained FDA approval, the first robotic adrenalectomy case reports appeared in the literature (5). It has been 20 years since the first robotic adrenalectomy, and almost 30 years since the first laparoscopic adrenalectomy. Recent data show that the numbers of adrenalectomies done in the United States with robotic assistance continues to rise, however laparoscopic adrenalectomy remains more common than robotic assisted adrenalectomy (6). It should be noted that these data are from the year 2012 as those were the most recently available nationwide data at 


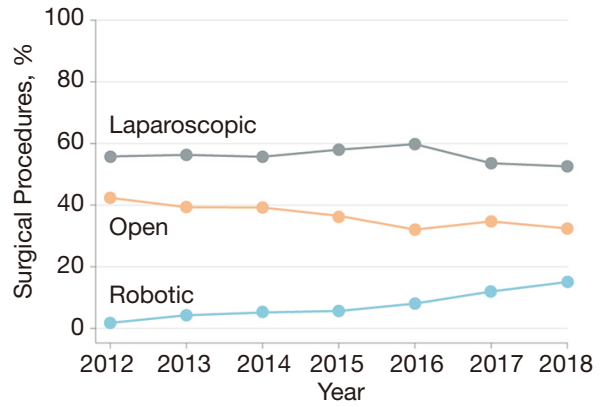

Figure 1 Temporal Trends in the Proportional Use of Robotic, Laparoscopic, and Open Surgery. Data are from the Michigan Surgical Quality Collaborative from January 1, 2012, through June 30,2018 (7). These data reflect practices at all hospitals included in the study.

the time of writing. It is clear that the number of roboticassisted surgeries has continued to rise in the period from 2012 to today (7). Regardless of the rising trends it is likely that laparoscopic adrenalectomy is still more common than robotic-assisted adrenalectomy, and it is reasonable to ask why robotic adrenalectomy has not surpassed laparoscopic adrenalectomy in the United States? There are several factors that may be associated with these trends.

According to the device manufacturers' data there were approximately 644,000 robotic-assisted surgical procedures performed in the United States in the year 2017. The United States performs more robotic-assisted operations than any other country in the world (8). In addition, the United States accounts for $65 \%$ of all DaVinci robotic machines installed and $73 \%$ of all robotic-assisted cases performed world-wide. However, despite the system being available since the year 2000, the majority of procedures performed with robotic-assistance were not general surgery procedures. In fact, it has been shown that by the year 2012, more than a decade after the introduction of the robotic platform, general surgery procedures accounted for only $1.8 \%$ of all robotic-assisted procedures. However, there was been a significant increase in adoption of the robot by general surgeons since 2012, such that between 2012 and 2018 there was a more than 8 fold increase in general surgery procedures done with robotic assistance (7) (see Figure 1). Adrenalectomy is interesting in this context because it is a procedure that is performed by both general surgeons as well as urologists in the United States. Data suggest that approximately $60 \%$ of adrenalectomies in the United States are performed by urologist and $40 \%$ by general surgeons (9). While urologists adopted the robot very early on after its introduction, it was nearly a decade before general surgeons began to use the robotic platform with any significant volume. It is likely that the numbers of adrenal surgeries done robotically remained lower than expected in this early period due in part to a lack of interest or enthusiasm on the part of general surgeons. It is possible that as general surgeons continue to adopt the robot, that more adrenalectomies will be performed robotically rather than laparoscopically. However, this may not necessarily hold true. This is because even though the number of general surgery procedures has risen steadily over the last decade, the majority of this rise in utilization of the robot is attributable to ventral and incisional hernia repairs in which there has been a nearly 45 -fold increase in the use of the robot for these procedures, representing a rise from $0.7 \%$ to $28.8 \%$ of ventral hernia procedures for example. In the same time period robotic-assisted complex cancer resections increased only 1.9 fold from $2.1 \%$ to $3.9 \%$ of all those particular procedures (7). Thus even though robotic surgery for general surgery procedures has risen rapidly, the majority of this increase in use is related to hernia procedures, thus the increasing use may not translate into increasing utilization of the robot for procedures like adrenalectomy. Based on these data alone it is likely that the numbers of robotic-assisted adrenalectomies will continue to rise in the United States as a function of increased access to the robot, but not at a rate as dramatic as the overall increasing trend of robot use in general.

There are many possible reasons why robotic adrenalectomy is not rising in popularity as rapidly as some other general surgery procedures. First, adrenalectomy is a low volume operation. According to data from the Nationwide Inpatient Sample there are approximately 6,000 adrenalectomies performed each year in the United States (10). In contrast, data from the same database estimates there are approximately 181,000 ventral hernia repairs performed per year in the United States which translates into a multi-billion dollar industry (11). As with any new technology marketing has played and continues to play an important role in the spread and adoption of robotic technology. Given that the market for adrenalectomies is dwarfed by so many other more common general surgical procedures it stands to reason that the majority of the marketing dollars spent on the robot is not on adrenalectomies.

Without extensive marketing dollars, expansion of robotic adrenalectomy is heavily dependent on availability of the 
robot, cost of the robot version of the procedure relative to alternatives, and the clinical advantages that the robot offers. As the robotic platform continues to be installed in more hospitals in the United States its availability continues to increase. A simple increase in availability of the robotic platform is thus driving some increase in robotic adrenalectomy cases. However, it is the combination of lack of marketing along with the cost-benefit analysis (perceived or real) that is likely keeping the use of the robot in adrenalectomy from exploding as it has done in hernia repair surgery in the recent past in the United States.

The cost-benefit analysis is essential to the success of robotic surgery in general, but it is particularly important for low volume operations such as adrenalectomy. In order for a technology like robotic adrenalectomy to be adopted surgeons must be willing to overcome the initial learning curve associated with using the robot. There are several costs associated with any surgical learning curve (8). An actual monetary cost, a cost in lost time, and a cost in surgeon stress. But even after the learning curve is surmounted it is generally accepted that in the United States there is some additional cost associated with robotic assisted cases when compared to their open or laparoscopic counterparts (7). Because of this, the clinical benefit associated with the robotic version of the operation has to outweigh all these additional costs in order for the robotic procedure to flourish. There are several papers in the literature comparing outcomes associated with robotic adrenalectomy $v s$. laparoscopic adrenalectomy and robotic adrenalectomy $v s$. open adrenalectomy. With the number and size of incisions being similar between the robotic and laparoscopic approach, robotic-assisted adrenalectomy is in many ways very similar to laparoscopic adrenalectomy. Any gains in robotic surgery compared to open surgery are also likely to be the same with laparoscopic surgery, thus findings in papers comparing robotic vs open adrenalectomy have limited value. Overall the main difference found between robotic and laparoscopic adrenalectomy is a decreased postoperative hospital stay (12). This has been shown in multiple studies, however while statistically significant these differences usually are not clinically relevant. In addition, some surgeons argue that the $3 \mathrm{D}$ view allows for improved dissection of the adrenal vein, and there is some evidence to suggest superior outcomes for robotic surgery in large adrenal tumors. Some surgeons have also argued that the wristed instruments also make it easier to do the fine dissection and ligation of the adrenal vein. Overall if there are any gains to be had with robotic-assisted adrenalectomy, those gains seem to be clinically minimal at best. Another way to view it is that the gains are not so great as to push many surgeons who are not familiar with the robot to begin performing robotic adrenalectomy. However, for a surgeon who is already facile with the robot, robotic-assisted adrenalectomy is a safe and feasible option and a likely easy conversion from straight laparoscopic surgery to roboticassisted surgery in this case. This seems to be the case, in that the majority of robotic assisted adrenal operations in the United States are performed by urologists as opposed to general surgeons (9). One area of improvement that is not talked about much in the literature is improvement in surgeon-related ergonomics. Laparoscopic adrenalectomy can be ergonomically challenging, particularly in obese patients. The position of the surgeon and assistant during the operation can be challenging, with stress placed on the wrists, knees and lower back of the surgeon. In this regard the robotic-assisted adrenalectomy is preferable, although quantifying this advantage is challenging.

\section{Conclusions}

There are more robotic-assisted cases performed in the United States than in any other country in the world, and the trend is toward increasing conversion from laparoscopic to robotic-assisted surgery. Currently, it appears that in the United States laparoscopic adrenalectomy remains more common than robotic adrenalectomy. However, there is a clear trend for increasing use of the robot in adrenalectomy, and the data suggest that in experienced hands there is no decrease in clinical outcomes with the robot. Given that the robotic version of the operation is equivalent or slightly better than the laparoscopic version, it is likely that in the United States as new generations of surgeons become familiar with the robot, and as the robot becomes more available, that a migration from laparoscopic to roboticassisted adrenalectomy will continue.

\section{Acknowledgments}

Funding: None.

\section{Footnote}

Provenance and Peer Review: This article was commissioned by the Guest Editor (Özer Makay) for the series "Robotic Adrenalectomy" published in Gland Surgery. The article was sent for external peer review organized by the Guest Editor 
and the editorial office.

Conflicts of Interest: The author has completed the ICMJE uniform disclosure form (available at http://dx.doi. org/10.21037/gs.2020.03.39). The series "Robotic Adrenalectomy" was commissioned by the editorial office without any funding or sponsorship. The author has no other conflicts of interest to declare.

Ethical Statement: The author is accountable for all aspects of the work in ensuring that questions related to the accuracy or integrity of any part of the work are appropriately investigated and resolved.

Open Access Statement: This is an Open Access article distributed in accordance with the Creative Commons Attribution-NonCommercial-NoDerivs 4.0 International License (CC BY-NC-ND 4.0), which permits the noncommercial replication and distribution of the article with the strict proviso that no changes or edits are made and the original work is properly cited (including links to both the formal publication through the relevant DOI and the license). See: https://creativecommons.org/licenses/by-nc-nd/4.0/.

\section{References}

1. Gagner M, Lacroix A, Bolté E. Laparoscopic adrenalectomy in Cushing's syndrome and pheochromocytoma. N Engl J Med 1992;327:1033.

2. Sardi A, McKinnon W. Laparoscopic adrenalectomy for primary aldosteronism. JAMA 1993;269:989-90.

Cite this article as: Grogan RH. Current Status of robotic adrenalectomy in the United States. Gland Surg 2020;9(3):840843. doi: $10.21037 /$ gs.2020.03.39
3. Smith CD, Weber CJ, Amerson JR. Laparoscopic adrenalectomy: new gold standard. World J Surg 1999;23:389-96.

4. Guidelines for the Minimally Invasive Treatment of Adrenal Pathology - A SAGES Publication [Internet]. [cited $2020 \mathrm{Feb}$ 19]. Available online: https://www.sages. org/publications/guidelines/guidelines-for-the-minimallyinvasive-treatment-of-adrenal-pathology/

5. Horgan S, Vanuno D. Robots in laparoscopic surgery. J Laparoendosc Adv Surg Tech A 2001;11:415-9.

6. Samreen S, Fluck M, Hunsinger M, et al. Laparoscopic versus robotic adrenalectomy: a review of the national inpatient sample. J Robot Surg 2019;13:69-75.

7. Sheetz KH, Claflin J, Dimick JB. Trends in the Adoption of Robotic Surgery for Common Surgical Procedures. JAMA Netw Open 2020;3:e1918911.

8. Childers CP, Maggard-Gibbons M. Estimation of the Acquisition and Operating Costs for Robotic Surgery. JAMA 2018;320:835-6.

9. Monn MF, Calaway AC, Mellon MJ, et al. Changing USA national trends for adrenalectomy: the influence of surgeon and technique. BJU Int 2015;115:288-94.

10. Murphy MM, Witkowski ER, Ng SC, et al. Trends in adrenalectomy: a recent national review. Surg Endosc 2010;24:2518-26.

11. Poulose BK, Beck WC, Phillips SE, et al. The chosen few: disproportionate resource use in ventral hernia repair. Am Surg 2013;79:815-8.

12. Economopoulos KP, Mylonas KS, Stamou AA, et al. Laparoscopic versus robotic adrenalectomy: A comprehensive meta-analysis. Int J Surg 2017;38:95-104. 